\title{
Pimonidazole Hydrochloride
}

National Cancer Institute

\section{Source}

National Cancer Institute. Pimonidazole Hydrochloride. NCI Thesaurus. Code C29366.

The hydrochloride salt form of pimonidazole, a hypoxia-selective 2-nitroimidazole with potential radiosensitizing activity. Pimonidazole is reduced in hypoxic environments such as those found in tumors. In hypoxic cells and tumors, reduced pimonidazole binds to thiol-containing proteins and glutathione. The resulting complexes accumulate in hypoxic tumors, deplete radioprotective thiol compounds, and cause the tumor to be more susceptible to radiation treatment. 